\title{
Surveillance of recent HIV infections among newly diagnosed HIV cases in Germany between 2008 and 2014
}

Alexandra Hofmann ${ }^{1,2^{*}}$, Andrea Hauser ${ }^{3}$, Ruth Zimmermann ${ }^{1}$, Claudia Santos-Hövener ${ }^{1}$, Jörg Bätzing-Feigenbaum ${ }^{4}$, Stephan Wildner ${ }^{5}$, Claudia Kücherer ${ }^{3}$, Norbert Bannert ${ }^{3}$, Osamah Hamouda ${ }^{6}$, Viviane Bremer ${ }^{1}$ and Barbara Bartmeyer ${ }^{1}$

\begin{abstract}
Background: The HIV surveillance system in Germany is based on mandatory, anonymous notification of newly diagnosed HIV cases by laboratories. Because the time between HIV infection and the diagnosis of HIV varies widely between persons, it is difficult to determine the number of cases of recent HIV infection among newly diagnosed cases of HIV. In Germany, the BED-capture-enzyme immunoassay (BED-CEIA) has been used to distinguish between recent and long-standing HIV infection. The aim of this analysis is to report the proportion of cases of recent HIV infection among newly diagnosed cases in Germany between 2008 and 2014 and to identify factors associated with recent infections.
\end{abstract}

Methods: A sample of voluntary laboratories among all HIV diagnostic laboratories was recruited. Residual blood from HIV diagnostic tests was spotted on filter paper as dried serum or dried plasma spots and was sent along with the notification form of the HIV cases. The BED-CEIA test was performed. A case was defined as recent HIV infection with a BED-CEIA test result of less than 0.8 normalized optical density, with the exclusion of CDC stage C. The proportion of recent newly diagnosed HIV infections among different groups (such as transmission groups, gender or age groups) was calculated. We used logistic regression to identify factors associated with recent HIV infection and to identify subpopulations with high proportions of recent HIV infections.

Results: Approximately 10,257 newly diagnosed cases were tested for recency using the BED-CEIA. In total, 3084 (30.4\%) of those were recently infected with HIV. The highest proportion of recent HIV infections was found among men who had sex with men (MSM) (35\%) and persons between 18 and 25 years of age (43. 0\%). Logistic regression revealed that female German intravenous drug users with a recent HIV infection had a higher chance of being detected than German MSM (OR 2.27).

Conclusions: Surveillance of recent HIV infection is a useful additional tool to monitor the HIV epidemic in Germany. We could observe ongoing HIV transmission in Germany in general and in different subgroups, and we could identify factors associated with recent HIV infection in Germany.

Keywords: Surveillance, HIV, Recent HIV infection, Test for recency of infection, Bed-CEIA, Germany

\footnotetext{
* Correspondence: HofmannA@rki.de

1 Department for Infectious Disease Epidemiology, Unit 34 HIV/AIDS, STI and

Blood-Borne Infections, Robert Koch-Institute, Seestr.10, 13353 Berlin,

Germany

${ }^{2}$ Charité, Universitätsmedizin, Berlin, Germany

Full list of author information is available at the end of the article
} 


\section{Background}

In 2014, 83,400 (77,000-91,200) people were estimated to be living with HIV/AIDS in Germany. The majority of them were men who have sex with men (MSM; approximately 53,800 ), followed by people who were infected heterosexually with HIV (HET; approximately 10,500) and approximately 7900 persons who were intravenous drug users (IDU). The estimated HIV epidemic in Germany peaked in 1985, followed by a decline in the 1990s, an increase from 2000 to 2006, and stable infection rates from 2006 to 2014. It was estimated by modelling that in 2014, approximately 3200 people were newly infected with HIV. The highest estimated number of HIV infections occurred among MSM (2300) [1].

In Germany, the HIV surveillance system is based on mandatory, anonymous notification of newly diagnosed HIV cases by laboratories. Additional epidemiological information regarding the HIV mode of transmission and other clinical data is reported by physicians. AIDS case reporting is voluntary [2]. In addition to HIV notification data, several long-term observational cohort studies in different HIV-positive populations, such as the study on the clinical surveillance of HIV disease [3], the German HIV seroconverter study [4] and other biological and behavioural studies in the most affected vulnerable groups have been performed.

The time between HIV infection and the diagnosis of HIV varies widely between individuals. Therefore, it is difficult to calculate the number of persons newly infected with HIV per year to determine the incidence of HIV infections by exclusively using newly diagnosed HIV cases. As a consequence, all other supportive sources, such as the HIV cohorts and the mortality register, are used to model the incidence of new HIV infections per year and the number of people who live with HIV/AIDS in Germany by using the imputation method [1, 5].

In addition to modelling an estimated incidence and prevalence of HIV infected persons in Germany, cases of newly diagnosed HIV are regularly tested serologically for recent HIV infection (TRI) since 2011 to improve the method of estimating the HIV epidemic in Germany and to identify populations with recent transmission of HIV. Numerous serological assays for TRI were developed and applied within the last decade [6-9]. Most of the tests are based on the measurement of the maturation of HIV1 specific antibodies, which occur within the first two years after seroconversion [10,11]. Accordingly, based on the increase of the antibody titres [12-14], the proportion of HIV-specific immunoglobulin G (IgG) antibodies relative to total IgG [15] or the avidity of antibodies [16-20], TRIs can distinguish between recently acquired and longstanding infections. For many years, the BED-captureenzyme immunoassay (BED-CEIA) [15] was the most commonly used recency assay [21-23]. The BED-CEIA was evaluated for Germany in a pilot study (2005-2007) [24] and finally applied in a nationwide cross-sectional incidence study $[25,26]$. The findings in this study were used to implement a nationwide surveillance system of recent HIV infections in 2011.

The aim of this analysis is to report the proportions of recent HIV infections among newly diagnosed HIV cases in Germany between 2008 and 2014 and to identify factors associated with recent infection in order to provide insight into current HIV transmission dynamics.

\section{Methods}

\section{Sample collection}

Newly diagnosed HIV cases were directly reported by laboratories to the Robert Koch Institute, RKI, which is the national public health institute of Germany. As the number of HIV notifications varies a lot between the reporting laboratories in Germany, only a subgroup of laboratories were recruited using convenience sampling for this analysis ( $n=108$; Fig. 1$)$. This subgroup of laboratories notified $90.5 \%$ of all newly HIV diagnosed cases between 2008 and 2014. These laboratories collected blood residuals from HIV diagnostics, which were spotted on filter paper (Whatman \# 903, GE Healthcare Bio-Science Corp, Westborough, USA) as dried serum spots (DSS) or dried plasma spots (DPS) for the surveillance of recent HIV infections. The DSS/DPS samples were sent together with the routine HIV notification form to the RKI. For this analysis, DSS/DPS samples were used from two different studies. They were collected between 1st March 2008 and 30th June 2010 for a nationwide cross-sectional study and between the 1st January 2011 and 31st December 2014 for the study "surveillance of recent HIV infections".

For this analysis, only persons were included, who were newly diagnosed with HIV between 2008 and 2014 and with one additional DSS/DPS sample available. HIV notifications and DSS/DPS samples were linked by using the number of the HIV notification form. Data on age, gender, mode of transmission, region of origin and assumed region of infection were used from the associated HIV notification form. Exclusion criteria for this analysis were unknown information on gender or age, age below 18 years, being infected with HIV via mother to child transmission, or DSS/DPS samples from double-notified HIV cases (Fig. 2). Ethical approval for the nationwide cross-sectional study was given by the ethics board at the Charité, University Medicine, Berlin, as well as approval from the data protection office of Germany according to the Federal Data Protection Act. The DSS/ DPS samples used were residuals from routine HIV diagnostic processing; therefore, no patient informed consent was given. Furthermore, the DSS/DPS samples 


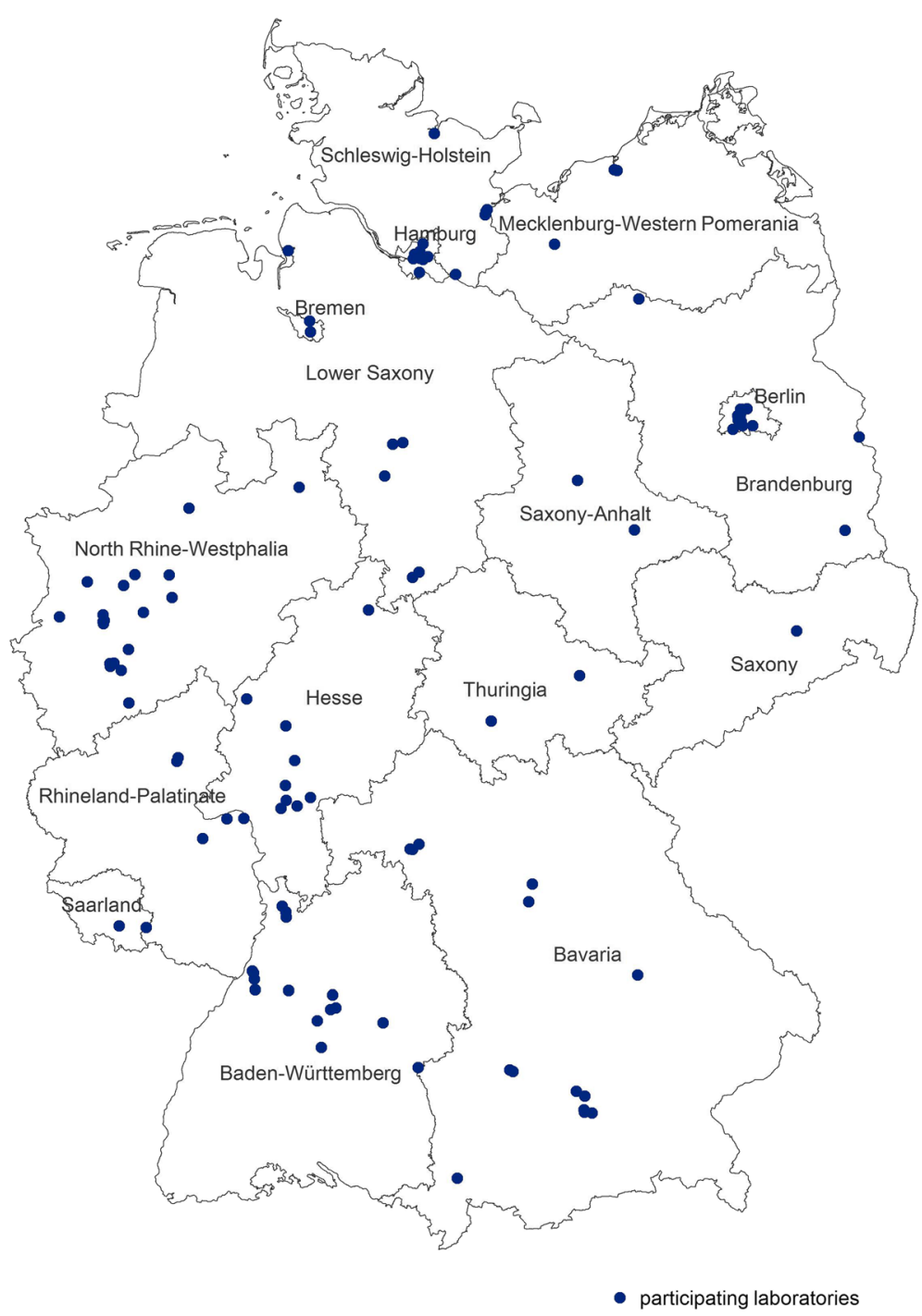

Fig. 1 Distribution of participating laboratories $(n=108)$ in Germany, 2008-2014. Map created with RegioGraph Analyse $\odot$ GfK GeoMarketing GmbH, Bruchsal, Germany

cannot be linked to an individual person because the HIV notification system is strictly anonymous. Additionally, the BED-CEIA is only licensed for epidemiological studies and not for individual diagnosis. Therefore, no extra benefit can be obtained by informing persons about positive BED-CEIA test results.

\section{Laboratory testing}

All DSS/DPS samples were tested for recency of HIV infection by the RKI study laboratory (HIV and Other Retroviruses Unit) using the BED-CEIA from the Calypte Biomedical Corporation, Portland, USA [27], and since 2014, the BED-CEIA from Sedia Biosciences Corporation, Portland, USA was used [28]. The BED-CEIA measures the proportion of HIV-1-specific immunoglobulin G (IgG) antibodies relative to total IgG at a normalized optical density (ODn). Cases were classified as recent HIV infection or long-standing infection by the quantitative output relative to a defined cut off of ODn $<0.8$. A recent HIV infection is determined, according to Sedia Biosciences Corporation, as an infection that occurred within the last 197 (range 127-236) days, or within the last 162 days for HIV subtype B [28]. Calypte reports a duration of 155 days [27], and in Germany, approximately 20 weeks (=140 days) was determined as the duration of a recent HIV infection [29]. The sensitivity and specificity of the BED-CEIA are low $(81.7 \%$; $89.1 \%)$ [15], and therefore, the test is recommended for surveillance purposes only using DSS/ DPS samples from individuals who have already been diagnosed with HIV.

To minimize false recent classification of the BEDCEIA, an additional step, the recent test algorithm (RITA), 


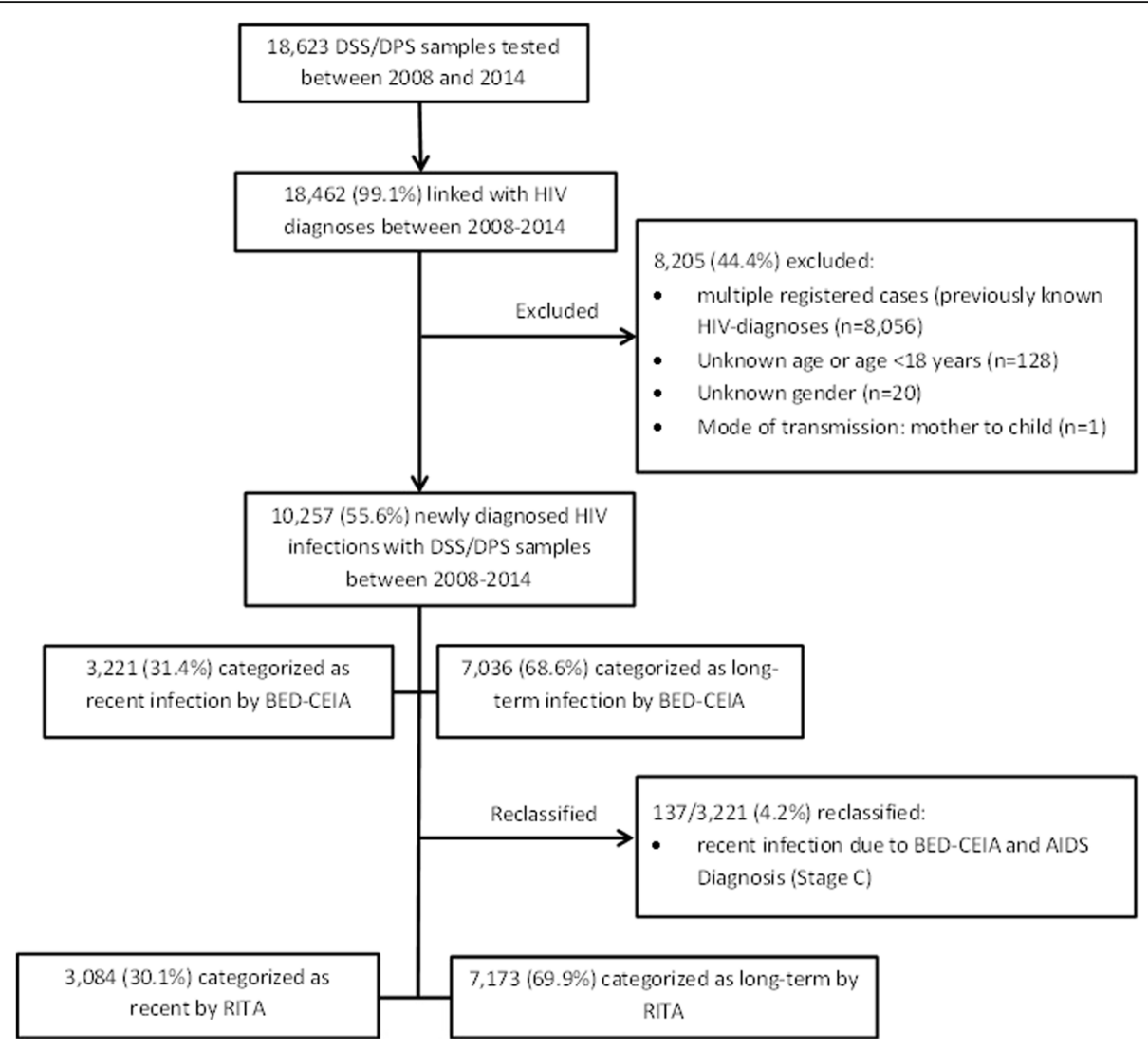

Fig. 2 Flowchart of DSS/DPS samples and HIV diagnoses included in the analyses between 2008 and 2014 in Germany. DSS/DPS: dried serum spots or dried plasma spots. RITA: recent test algorithm. BED-CEIA: BED-capture-enzyme immunoassay

was applied following the European Centre for Disease Prevention and Control (ECDC) recommendation [30]. It is recommended that cases with a recent BED-CEIA test result should be recoded as long-standing infection if the case was reported with an AIDS defining illness (stage $\mathrm{C}$ of the Centers for Disease Control and Prevention (CDC) criteria), CD4 cell counts $<200$ cells $/ \mathrm{mm}^{3}$, or a reported plasma viral load below 400 copies/ml. Data on CD4 cell counts and viral load are often missing in the HIV notification form and are therefore scarce. For example, in 2014, only 34\% of the newly diagnosed HIV cases had information regarding CD4 cell count [31]. Therefore, only the information about AIDS defining illnesses reported on the notification form was used to minimize false BED-CEIA test results.

A newly diagnosed case of recent infection with HIV is therefore defined as a BED-CEIA test result with a cut off of less than 0.8 ODn and without any documented AIDS defining illnesses. In contrast, a case with longstanding HIV infection is defined as a case in which the BED-CEIA test result has a cut off equal to or higher than 0.8 ODn or an AIDS defining illness. By using this method, cases with a false recent test result can be identified and corrected; however false long-standing (FLS) test results were not corrected. To correct the FLS test results, as well, further information about the cases is needed, such as previously documented HIV RNA+/Abor p24Ag+/Ab- within 180 days of the sample date, clinical symptoms of seroconversion, or previously documented HIV-negative test result within 180 days of the sample date [30]. This information is not available on the HIV notification form.

\section{Statistical analysis}

Analyses were performed using STATA 13.0 (Stata Statistical Software: Release 13, United States). To guarantee comparability between newly diagnosed HIV cases with DSS/DPS and all newly diagnosed HIV cases, a weight has been calculated by dividing the proportion of variable specifications among all newly diagnosed cases with the proportion of variable specifications of those newly diagnosed cases with DSS/DPS.

We analysed the number and the proportion of recent HIV infections among cases using the chi ${ }^{2}$ test for bivariate comparison and logistic regression to assess the odds ratio for recent infections. Univariate logistic regression was performed. Factors with significant odds ratios were included in the multivariable logistic regression. Time trends were analysed by using logistic regression with recent infection 
as a dependent variable and diagnosed cases with DSS/DPS per year as an independent variable. Multivariable logistic regression with recent infection as a dependent variable was also used to identify subgroups according to their gender and origin within different modes of transmission. Unknown data was included into analysis, such as unknown mode of transmission, origin or assumed region of infection for identifying subgroups. For the proportion of recent infections, the $95 \%$ confidence interval $(95 \% \mathrm{CI})$ was calculated. A Mann-Whitney (Wilcoxon) rank sum test of medians was used to compare the age of those with recent infection and those with long-standing infection.

\section{Results}

Participating laboratories and availability of DSS/DPS among newly diagnosed HIV cases

Between 2008 and 2014, a total of 39,170 HIV diagnoses were reported by 227 laboratories nationwide. The participating laboratories $(n=108 / 227 ; 47.6 \%)$ reported approximately $91.3 \%(n=35,756)$ of all HIV diagnoses between 2008 and 2014. Of these 39,170 HIV diagnoses, $20,896(53.3 \%)$ were newly diagnosed cases (double or unknown notifications were excluded from analysis). During the same time period, a total of 18,623 DSS/DPS samples were sent to the RKI by these laboratories and 99.1\% could be linked to an HIV notification form (Fig. 2). The distribution of the numbers of reported HIV cases by laboratories within subgroups is similar for newly diagnosed HIV cases with DSS/DPS $(n=10,257)$ and all newly diagnosed cases $(n=20,487$ Table 1$)$. The different weights ranged between 0.9 and 1.1 (Median 1), therefore no additional weight adjustment was included. Especially, the number of collected DSS/DPS samples differed in 2010 $(n=4.0)$, when DSS/DPS samples were collected for a shorter time period than in other years. The number of collected DSS/DPS samples increased significantly over time $\left(\operatorname{chi}^{2} p<0.001\right)$.

\section{Characteristics of the overall study population of newly diagnosed HIV cases with DSS/DPS samples between 2008 and 2014}

The study population consisted mostly of men $(83.3 \% ; n$ $=8,541 / 10,257)$. The main mode of HIV transmission was MSM (58.4\%), followed by an unknown mode of transmission (20.0\%), HET (18.6\%) and IDU (3.0\%). One third (33.6\%) of the study population was between 25 and 34 years old (Table 2). Most patients originated from Germany (62.4\%), followed by people from subSaharan Africa (9.5\% (Table 3). The most frequently reported presumed region of infection was Germany (69.5\%), followed by sub-Saharan Africa (7.2\%) (Table 3).

Information about $\mathrm{CD} 4$ cell count among newly diagnosed HIV cases with DSS/DPS was available from 32.1\% ( $n=3297$ ). Of those cases, $51.6 \%$ showed CD4 cell
Table 1 Comparison of newly diagnosed HIV cases with and without DSS/DPS samples by gender, origin, age, mode of transmission, region of presumed infection, region of origin and year of diagnosis, 2008-2014, Germany

\begin{tabular}{|c|c|c|c|c|c|}
\hline \multirow[b]{3}{*}{ Total } & \multicolumn{5}{|c|}{ Newly diagnosed HIV cases, n \% } \\
\hline & \multicolumn{2}{|l|}{ total } & \multicolumn{2}{|c|}{ with DSS/DPS ${ }^{4}$ Sample } & \multirow[t]{2}{*}{ weighting } \\
\hline & 20,487 & $100 \%$ & 10,257 & $100 \%$ & \\
\hline \multicolumn{6}{|l|}{ Gender } \\
\hline Men & 17,137 & $83.6 \%$ & 8,541 & $83.3 \%$ & 1.0 \\
\hline Women & 3,350 & $16.4 \%$ & 1,716 & $16.7 \%$ & 1.0 \\
\hline \multicolumn{6}{|l|}{ Region of origin } \\
\hline Germany & 12,830 & $62.6 \%$ & 6,405 & $62.4 \%$ & 1.0 \\
\hline Abroad & 5,454 & $26.6 \%$ & 2,853 & $27.8 \%$ & 1.0 \\
\hline Unknown & 2,203 & $10.8 \%$ & 999 & $9.7 \%$ & 1.1 \\
\hline \multicolumn{6}{|l|}{ Age group } \\
\hline$<25$ years & 2,298 & $11.2 \%$ & 1,216 & $11.9 \%$ & 0.9 \\
\hline 25-34 years & 6,687 & $32.6 \%$ & 3,443 & $33.6 \%$ & 1.0 \\
\hline $35-44$ years & 5,999 & $29.3 \%$ & 2,986 & $29.1 \%$ & 1.0 \\
\hline $45-54$ years & 3,824 & $18.7 \%$ & 1,838 & $17.9 \%$ & 1.0 \\
\hline$>=55$ years & 1,679 & $8.2 \%$ & 774 & $7.5 \%$ & 1.1 \\
\hline \multicolumn{6}{|c|}{ Mode of transmission } \\
\hline $\mathrm{MSM}^{1}$ & 11,541 & $56.3 \%$ & 5,993 & $58.4 \%$ & 1.0 \\
\hline $\mathrm{HET}^{2}$ & 3,789 & $18.5 \%$ & 1,909 & $18.6 \%$ & 1.0 \\
\hline $\mathrm{IDU}^{3}$ & 649 & $3.2 \%$ & 307 & $3.0 \%$ & 1.1 \\
\hline Unknown & 4,508 & $22.0 \%$ & 2,048 & $20.0 \%$ & 1.1 \\
\hline \multicolumn{6}{|c|}{ Region of presumed infection } \\
\hline Germany & 14,184 & $69.2 \%$ & 7,124 & $69.5 \%$ & 1.0 \\
\hline Abroad & 3,506 & $17.1 \%$ & 1,803 & $17.6 \%$ & 1.0 \\
\hline Unknown & 2,797 & $13.7 \%$ & 1,330 & $13.0 \%$ & 1.1 \\
\hline \multicolumn{6}{|c|}{ Year of Diagnosis } \\
\hline 2008 & 2,745 & $13.4 \%$ & 1,264 & $12.3 \%$ & 1.1 \\
\hline 2009 & 2,815 & $13.7 \%$ & 1,335 & $13.0 \%$ & 1.1 \\
\hline $2010^{a}$ & 2,653 & $12.9 \%$ & 332 & $3.2 \%$ & 4.0 \\
\hline 2011 & 2,644 & $12.9 \%$ & 1,624 & $15.8 \%$ & 0.8 \\
\hline 2012 & 2,920 & $14.3 \%$ & 1,779 & $17.3 \%$ & 0.8 \\
\hline 2013 & 3,232 & $15.8 \%$ & 1,890 & $18.4 \%$ & 0.9 \\
\hline 2014 & 3,478 & $17.0 \%$ & 2,033 & $19.8 \%$ & 0.9 \\
\hline
\end{tabular}

${ }^{1}$ MSM men who have sex with men

${ }^{2}$ IDU persons who are intravenous drug users

${ }^{3}$ HET persons with heterosexual contact

${ }^{4}$ DSS/DPS Sample Dried Serum Spot/Dried Plasma Spot

${ }^{a}$ DSS/DPS samples were not continuously collected due to the end of the first study in June 2010

counts below 350 cells $(n=1700)$. Late presentation (CD4 cell counts $<350$ cells $/ \mathrm{mm}^{3}$ or CDC stadium AIDS) was identified in $10.1 \%(n=949 / 9363)$ of newly diagnosed cases with DSS/DPS with available data. Virus load information was available for $36.6 \%$ ( $n=3755$ / $10,257)$ of newly diagnosed HIV cases with DSS/DPS. 
Table 2 Characteristics and factors associated with recent HIV infection among newly diagnosed HIV cases using DSS/DPS samples in Germany, 2008-2014

\begin{tabular}{|c|c|c|c|c|c|c|c|c|}
\hline \multirow{3}{*}{$\begin{array}{l}\text { Factor } \\
\text { Total }\end{array}$} & \multicolumn{4}{|c|}{ Newly diagnosed HIV cases, n \% } & \multicolumn{2}{|c|}{ Univariate analysis } & \multicolumn{2}{|c|}{ Multivariable analysis $(n=10,257)$} \\
\hline & \multicolumn{2}{|c|}{ total with DSS/DPS ${ }^{4}$} & \multicolumn{2}{|c|}{ with recent infection } & \multirow[t]{2}{*}{$O R^{*}$} & \multirow[t]{2}{*}{$95 \% \mathrm{Cl}^{\circ}$} & \multirow[t]{2}{*}{$\mathrm{OR}^{*}$} & \multirow[t]{2}{*}{$95 \% \mathrm{Cl}^{\circ}$} \\
\hline & 10,257 & $100 \%$ & 3,084 & $30.01 \%$ & & & & \\
\hline \multicolumn{9}{|l|}{ Gender } \\
\hline Men & 8,541 & $83.3 \%$ & 2,705 & $31.7 \%$ & (ref) & & (ref) & \\
\hline Women & 1,716 & $16.7 \%$ & 379 & $22.1 \%$ & $0.61 \#$ & $0.54-0.69$ & 0.94 & $0.79-1.12$ \\
\hline \multicolumn{9}{|l|}{ Region of origin } \\
\hline Germany & 6,405 & $62.5 \%$ & 2,121 & $33.1 \%$ & (ref) & & (ref) & \\
\hline Abroad & 2,853 & $27.8 \%$ & 673 & $23.6 \%$ & $0.62 \#$ & $0.56-0.69$ & $0.74 \#$ & $0.66-0.84$ \\
\hline Unknown & 999 & $9.7 \%$ & 290 & $29.0 \%$ & 0.83\#\# & $0.71-0.96$ & 0.94 & $0.80-1.11$ \\
\hline \multicolumn{9}{|l|}{ Age group } \\
\hline$<25$ years & 1,216 & $11.9 \%$ & 523 & $43.0 \%$ & (ref) & & (ref) & \\
\hline 25-34 years & 3,443 & $33.6 \%$ & 1,091 & $31.7 \%$ & $0.61 \#$ & $0.54-0.70$ & $0.63 \#$ & $0.55-0.72$ \\
\hline $35-44$ years & 2,986 & $29.1 \%$ & 846 & $28.3 \%$ & $0.52 \#$ & $0.46-0.60$ & $0.53 \#$ & $0.46-0.61$ \\
\hline $45-54$ years & 1,838 & $17.9 \%$ & 470 & $25.6 \%$ & $0.46 \#$ & $0.39-0.53$ & $0.45 \#$ & $0.39-0.53$ \\
\hline$>=55$ years & 774 & $7.5 \%$ & 154 & $19.9 \%$ & $0.33 \#$ & $0.27-0.41$ & $0.35 \#$ & $0.28-0.43$ \\
\hline \multicolumn{9}{|c|}{ Mode of transmission } \\
\hline MSM $^{1}$ & 5,993 & 58.4 & 2,095 & $35.0 \%$ & (ref) & & (ref) & \\
\hline $\mathrm{HET}^{2}$ & 1,909 & 18.6 & 393 & $20.6 \%$ & $0.48 \#$ & $0.43-0.55$ & $0.65 \#$ & $0.54-0.79$ \\
\hline $\mathrm{IDU}^{3}$ & 307 & 3.0 & 107 & $34.9 \%$ & 1.00 & $0.78-1.27$ & 1.18 & $0.92-1.51$ \\
\hline Unknown & 2,048 & 20.0 & 489 & $23.9 \%$ & $0.58 \#$ & $0.52-0.65$ & $0.68 \#$ & $0.60-0.77$ \\
\hline \multicolumn{9}{|c|}{ Region of presumed infection } \\
\hline Germany & 7,124 & $69.4 \%$ & 2,346 & $32.9 \%$ & (ref) & & (ref) & \\
\hline Abroad & 1,803 & $17.6 \%$ & 387 & $21.5 \%$ & $0.56 \#$ & $0.49-0.63$ & 0.85\#\# & $0.73-0.99$ \\
\hline Unknown & 1,330 & 13.0 & 351 & $26.4 \%$ & $0.73 \#$ & $0.64-0.83$ & 0.98 & $0.84-1.14$ \\
\hline
\end{tabular}

${ }^{1}$ MSM: men who have sex with men

${ }^{2}$ IDU: persons who are intravenous drug users

${ }^{3} \mathrm{HET}$ : persons with heterosexual contact

${ }^{4}$ DSS/DPS: Dried Serum Spot/Dried Plasma Spot

*OR: odds ratio

$95 \%$ Cl: $95 \%$ Confidence interval

$\# p$-value: $p<=0.001$

\#\# $p$-value: $p<=0.05$

\section{Proportion of recent HIV infections among newly diagnosed HIV cases with DSS/DPS samples between 2008 and 2014}

In total, 3221 (31.4\%; 95\% CI 30.5-32.2\%) cases were classified as recent infections, of which 137 (4.2\%) were reclassified according to the ECDC recommendation (see Fig. 2) [30]. The final study population consisted of $30.1 \%$ of cases with recent infection $(n=3084$; $95 \% \mathrm{CI}$ 29.2-31.0\%). The proportion of recent HIV infections among men was $31.7 \%$ compared to $22.1 \%$ among women $\left(\mathrm{chi}^{2} p<0.001\right)$. The highest proportion of recent infections (43.0\%) was found among young persons between 18 and 25 years of age (Table 2). Within the different modes of transmission, the highest proportion of recent infections was found among MSM (35.0\%), followed by IDU (34.9\%) (Table 2). Persons with a recent
HIV infection were significantly younger than those with a long-standing HIV infection (median age 34 vs. 37 years; $p<0.001$ Wilcoxon). MSM with recent HIV infection were significantly younger than MSM with a long-standing HIV infection (median age 33 vs. 36 years; $p<0.001$ Wilcoxon). No difference in age regarding recent HIV infection could be found for HET (median age 35 vs. $36 ; p=0.1215$ Wilcoxon) and IDU (median age 34 vs. $36 ; p=0.0990$ Wilcoxon).

The proportion of recent infection was 33.1\% among Germans and 17.3\% among sub-Saharan Africans (Table 3). Newly diagnosed HIV cases from Germany were more likely to be recent infected than those identified among newly diagnosed HIV cases originating from abroad (33.1\% vs. $23.6 \%$; chi $^{2} p<0.001$ ) (Table 2). Persons reported to be infected with HIV in the Western Europe 
Table 3 Origin and presumed region of infection in cases of recent HIV infection among newly diagnosed HIV cases using DSS/DPS samples in Germany, 2008-2014

\begin{tabular}{|c|c|c|c|c|c|c|}
\hline \multirow{3}{*}{ Region of origin } & \multicolumn{4}{|c|}{ Newly diagnosed HIV cases, n \% } & \multicolumn{2}{|c|}{$\underline{\text { Univariate analysis }}$} \\
\hline & \multicolumn{2}{|c|}{ Total with DSS/DPS ${ }^{1}$} & \multicolumn{2}{|c|}{ With recent infection } & \multirow[t]{2}{*}{$\mathrm{OR}^{*}$} & \multirow[t]{2}{*}{$95 \% \mathrm{Cl}^{\circ}$} \\
\hline & & & & & & \\
\hline Germany & 6,405 & $62.4 \%$ & 2,121 & $33.1 \%$ & (ref) & \\
\hline Eastern Europe & 384 & 3.7 & 90 & $23.4 \%$ & $0.62 \#$ & $0.49-0.79$ \\
\hline Western Europe & 310 & 3.0 & 98 & $31.6 \%$ & 0.93 & $0.73-1.19$ \\
\hline Central Europe & 464 & 4.5 & 143 & $30.8 \%$ & 0.90 & $0.73-1.10$ \\
\hline Asia / Oceania & 274 & 2.7 & 65 & $23.7 \%$ & $0.63 \#$ & $0.47-0.83$ \\
\hline America / Caribbean & 265 & 2.6 & 68 & $25.7 \%$ & $0.70 \# \#$ & $0.53-0.92$ \\
\hline North / Northeast Africa & 90 & 0.9 & 20 & $22.2 \%$ & $0.58 \# \#$ & $0.35-0.95$ \\
\hline sub-Saharan Africa & 978 & 9.5 & 169 & 17.3 & $0.42 \#$ & $0.35-0.50$ \\
\hline Unknown region & 88 & 0.9 & 20 & $22.7 \%$ & $0.59 \# \#$ & $0.36-0.98$ \\
\hline Unknown & 999 & 9.7 & 290 & $29.0 \%$ & $0.83 \# \#$ & $0.71-0.96$ \\
\hline \multicolumn{7}{|l|}{ Region of origin (combined) } \\
\hline Germany & 6,405 & 62.4 & 2,121 & $33.1 \%$ & (ref) & \\
\hline Abroad & 2,853 & 27.8 & 673 & $23.6 \%$ & $0.62 \#$ & $0.56-0.69$ \\
\hline Unknown & 999 & 9.7 & 290 & $29.0 \%$ & $0.83 \# \#$ & $0.71-0.96$ \\
\hline \multicolumn{7}{|l|}{ Region of presumed infection } \\
\hline Germany & 7,124 & 69.5 & 2,346 & $32.9 \%$ & (ref) & \\
\hline Eastern Europe & 215 & 2.1 & 41 & $19.1 \%$ & $0.48 \#$ & $0.34-0.68$ \\
\hline Western Europe & 205 & 2.0 & 70 & $34.1 \%$ & 1.06 & $0.79-1.42$ \\
\hline Central Europe & 145 & 1.4 & 36 & $24.8 \%$ & $0.67 \# \#$ & $0.46-0.98$ \\
\hline Asia / Oceania & 266 & 2.6 & 54 & $20.3 \%$ & $0.52 \#$ & $0.39-0.70$ \\
\hline America / Caribbean & 128 & 1.2 & 30 & $23.4 \%$ & $0.62 \# \#$ & $0.41-0.94$ \\
\hline North / Northeast Africa & 43 & 0.4 & 13 & 30.2 & 0.88 & $0.46-1.70$ \\
\hline sub-Saharan Africa & 737 & 7.2 & 129 & $17.5 \%$ & $0.43 \#$ & $0.36-0.53$ \\
\hline Unknown Region & 64 & 0.6 & 14 & $21.9 \%$ & 0.57 & $0.31-1.03$ \\
\hline Unknown & 1,330 & 13.0 & 351 & $26.4 \%$ & $0.73 \#$ & $0.63-0.83$ \\
\hline \multicolumn{7}{|c|}{ Region of presumed infection (combined) } \\
\hline Germany & 7,124 & 69.5 & 2,346 & $32.9 \%$ & (ref) & \\
\hline Abroad & 1,803 & 17.6 & 387 & $21.5 \%$ & $0.56 \#$ & $0.49-0.63$ \\
\hline Unknown & 1,330 & 13.0 & 351 & $26.4 \%$ & $0.73 \#$ & $0.64-0.83$ \\
\hline
\end{tabular}

${ }^{\circ} 95 \%$ Cl: $95 \%$ confidence interval

*OR: odds ratio

1 DSS/DPS: Dried Serum Spot/Dried Plasma Spot

$\# p$-value: $p<=0.001$

$\#$ \# $p$-value: $p<=0.05$

region (excluding Germany) had the highest proportion of recent infections (34.1\%), followed by people from Germany (32.9\%) as the presumed region of infection (Table 3). Cases with a recent HIV infection were more likely infected with HIV in Germany than abroad (32.9\% compared to $21.5 \%$ chi $\left.^{2} p<0.001\right)$. Cases from abroad with a recent infection were more likely reported to have acquired the infection in Germany $\left(30.8 \%\right.$ vs. $18.5 \%$; chi $^{2}$ $p<0.001)$. Cases with recent infection and available information on CD4 cell showed significantly higher CD4 cell counts than cases with long standing infection (Median 485 vs. $252 \mathrm{CD} 4$ cells $/ \mathrm{mm}^{3} ; p<0.001$ Wilcoxon). Among $2.2 \%$ ( $n=21 / 949)$ of cases defined as late presenters by $\mathrm{CD} 4$ cell count or CDC stage, a recent infection was identified by using the BED-CEIA.

\section{Proportion of recent infections over time within transmission groups}

The newly diagnosed HIV cases with available DSS/DPS samples increased from 1264 in 2008 to 2033 in 2014. 
The DSS/DPS sample collection decreased in June 2010 due to the end of the first study (Table 1). The proportion of recent HIV infections per year varied from $26 \%$ in 2010 up to $32 \%$ in 2012, with an overall increase of $4 \%$ between 2008 and 2014 (data not shown). The overall increase could be confirmed by univariate logistic regression (OR 1.023; 95\% CI 1.006-1.049; $p<0.012$ ). A similar trend was observed for MSM (OR 1.030; 95\% CI 1.003-1.058) and for HET (OR 1.079; 95\% CI 1.021.140). No significant trend over time (OR 0.965; 95\% CI $0.865-1.076 ; p<0.521$ ) was seen in IDU (Fig. 3).

\section{Association between recent infections and other variables} The univariate logistic regression revealed that several factors were associated with recent HIV infection, such as mode of transmission, age, gender and region of infection, which are shown in Table 2. These associations were confirmed by multivariable logistic regression, which showed that cases with a different mode of transmission than MSM were more likely to have a longstanding HIV infection. Including persons younger than 25 years, the proportion of persons with long-standing HIV infections increased with age (Table 2).

An additional multivariable logistic regression aimed to identify sub-groups within the main transmission groups revealed, that female German intravenous drug users had a higher chance of being diagnosed with a recent HIV infection (OR 2.49; 95\% CI 1.40-4.41) than German MSM (Table 4). However, the numbers of female German drug users were small, with 28 (57.1\%) cases of recent HIV infection among 49 female German drug users in total. Other groups with a lower chance of being recently infected with HIV compared to German MSM were German heterosexual females (OR 0.70; 95\% CI 0.55-0.90), heterosexual females with an origin abroad (OR 0.44; 95\% CI 0.35-0.54), heterosexual females with an unknown origin (OR 0.34; 95\% CI 0.17-0.70) and heterosexual men with an origin abroad (OR $0.39,95 \% \mathrm{CI}$ 0.29-0.53) (Table 4).

\section{Discussion}

This was the first analysis in Germany on national level to determine the proportion of recent HIV infections among newly diagnosed HIV cases by using the BEDCEIA according to ECDC corrections. One-third of newly diagnosed HIV infections between 2008 and 2014 were recent infections within the six months before diagnosis. Overall, we found a slight but significant increasing trend of recent HIV infection between 2008 and 2014, particularly among MSM and HET. The highest proportion of recent HIV infections was found in MSM (35.0\%) in contrast to a low proportion in HET (20.6\%). Logistic regression identified that only the small group of female German IDU had a higher chance of being newly diagnosed and coincidentally infected recently with HIV than MSM.

The overall proportion of recent HIV infections in Germany is similar to that of other European countries using the BED-CEIA for TRI. The overall proportion of recent HIV infections ranged from $23 \%$ among newly diagnosed cases in Catalonia, Spain (2006-2008) [32] to $35 \%$ in Sweden (2003-2010) [33]. However, in countries using other TRI, such as England, Northern Ireland, Wales or France, the proportion of recent HIV

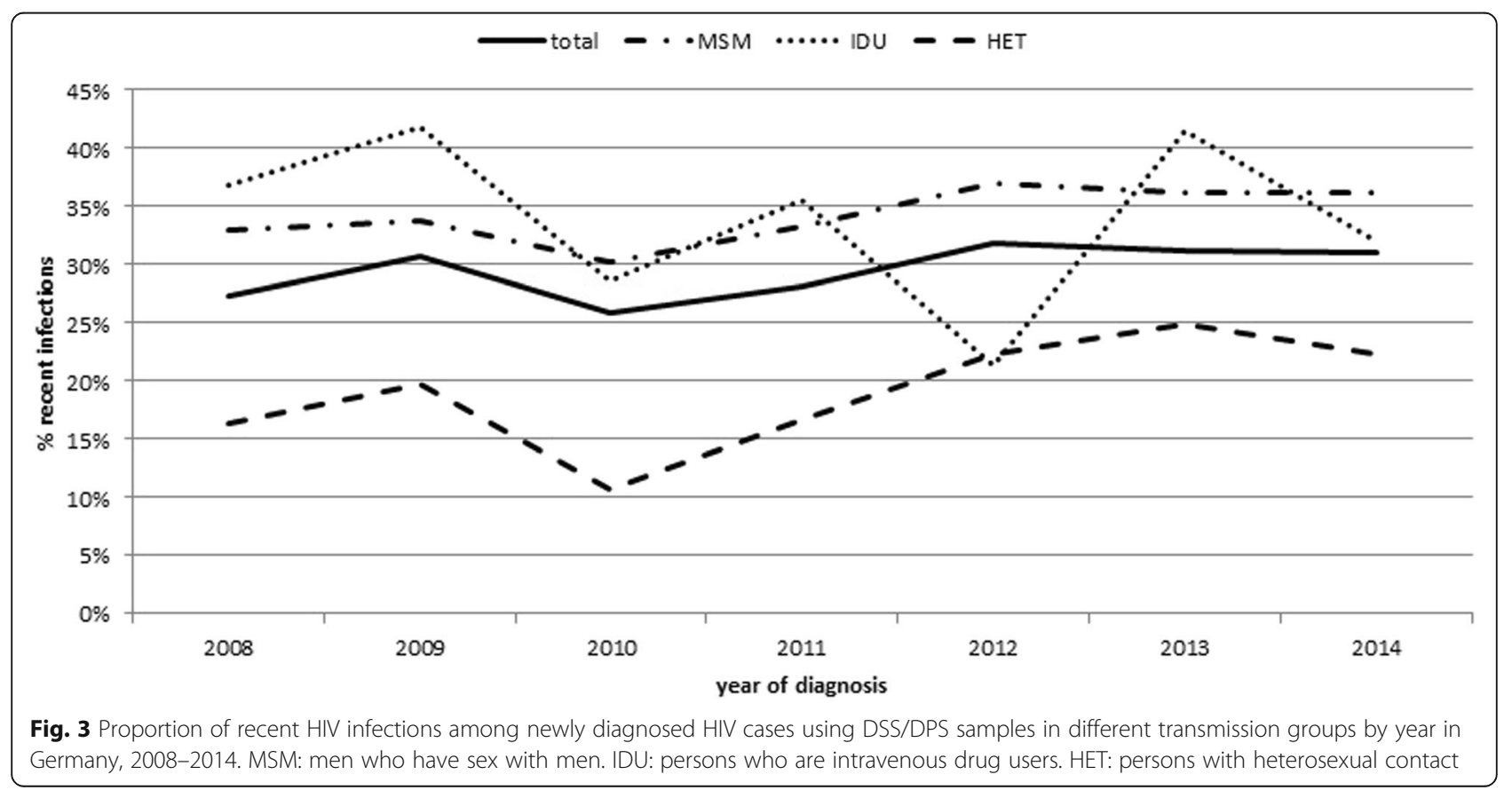


Table 4 Characteristics of subpopulations within the different modes of transmission with recent HIV infection among newly diagnosed HIV cases with DSS/DPS samples in Germany, 2008-2014

\begin{tabular}{|c|c|c|c|c|c|c|c|c|c|}
\hline \multirow{2}{*}{\multicolumn{3}{|c|}{ Subpopulations }} & \multicolumn{3}{|c|}{ Newly diagnosed HIV cases, n \% } & \multicolumn{2}{|c|}{ Univariate analysis } & \multicolumn{2}{|c|}{ Multivariable analysis $(n=10,257)$} \\
\hline & & & \multirow{2}{*}{$\frac{\text { Total }}{4,654}$} & \multicolumn{2}{|c|}{ With recent infection } & \multirow{2}{*}{$\frac{O R^{*}}{\text { (ref) }}$} & \multirow[t]{2}{*}{$95 \% \mathrm{Cl}^{\circ}$} & \multirow{2}{*}{$\frac{O R^{*}}{\text { (ref) }}$} & \multirow[t]{2}{*}{$95 \% \mathrm{Cl}^{\circ}$} \\
\hline $\mathrm{MSM}^{1}$ & Men & German Origin & & 1,652 & $35.5 \%$ & & & & \\
\hline & & non-German Origin & 944 & 316 & $33.5 \%$ & 0.91 & $0.79-1.06$ & 0.87 & $0.75-1.02$ \\
\hline & & Unknown & 395 & 127 & $32.2 \%$ & 0.86 & $0.69-1.07$ & 0.85 & $0.68-1.07$ \\
\hline \multirow[t]{6}{*}{$\mathrm{IDU}^{2}$} & Men & German Origin & 92 & 37 & $40.2 \%$ & 1.22 & $0.80-1.86$ & 1.35 & $0.88-2.06$ \\
\hline & & non-German Origin & 114 & 26 & $22.8 \%$ & $0.54 \# \#$ & $0.35-0.83$ & $0.58 \# \#$ & $0.37-0.91$ \\
\hline & & Unknown & 24 & 7 & $29.2 \%$ & 0.75 & $0.31-1.81$ & 0.76 & $0.31-1.85$ \\
\hline & Women & German Origin & 49 & 28 & $57.1 \%$ & 2.42\#\# & $1.37-4.28$ & 2.49\#\# & $1.40-4.41$ \\
\hline & & non-German Origin & 17 & 4 & $23.5 \%$ & 0.56 & $0.18-1.72$ & 0.52 & $0.17-1.61$ \\
\hline & & Unknown & 11 & 5 & $45.5 \%$ & 1.51 & $0.46-4.97$ & 1.50 & $0.45-4.99$ \\
\hline \multirow[t]{6}{*}{$\mathrm{HET}^{3}$} & Men & German Origin & 175 & 49 & $28.0 \%$ & $0.71 \# \#$ & $0.51-0.99$ & 0.96 & $0.68-1.36$ \\
\hline & & non-German Origin & 414 & 66 & $15.9 \%$ & $0.34 \#$ & $0.26-0.45$ & $0.39 \#$ & $0.29-0.53$ \\
\hline & & Unknown & 13 & 6 & $46.2 \%$ & 1.56 & $0.52-0.46$ & 1.92 & $0.63-5.80$ \\
\hline & Women & German Origin & 347 & 95 & $27.4 \%$ & 0.69\#\# & $0.54-0.87$ & 0.70\#\# & $0.55-0.90$ \\
\hline & & non-German Origin & 901 & 168 & $18.6 \%$ & $0.42 \#$ & $0.35-0.50$ & $0.44 \#$ & $0.35-0.54$ \\
\hline & & Unknown & 59 & 9 & $15.3 \%$ & 0.33\#\# & $0.16-0.67$ & 0.34\#\# & $0.17-0.70$ \\
\hline \multirow[t]{6}{*}{ Unknown } & Men & German Origin & 960 & 236 & $24.6 \%$ & $0.59 \#$ & $0.51-0.69$ & $0.67 \#$ & $0.57-0.79$ \\
\hline & & non-German Origin & 351 & 67 & $19.1 \%$ & $0.43 \#$ & $0.33-0.56$ & $0.48 \#$ & $0.36-0.63$ \\
\hline & & Unknown & 405 & 116 & $28.6 \%$ & $0.73 \# \#$ & $0.58-0.91$ & 0.80 & $0.62-1.03$ \\
\hline & Women & German Origin & 128 & 24 & $18.8 \%$ & $0.42 \#$ & $0.27-0.66$ & $0.46 \#$ & $0.30-0.73$ \\
\hline & & non-German Origin & 112 & 26 & $23.2 \%$ & $0.58 \# \#$ & $0.35-0.86$ & $0.58 \# \#$ & $0.37-0.91$ \\
\hline & & Unknown & 92 & 20 & $21.7 \%$ & $0.50 \# \#$ & $0.31-0.83$ & $0.54 \# \#$ & $0.32-0.90$ \\
\hline \multicolumn{10}{|c|}{ Region of presumed infection } \\
\hline \multicolumn{3}{|c|}{ Germany } & 7,124 & 2,346 & $32.9 \%$ & (ref) & & (ref) & \\
\hline \multicolumn{3}{|l|}{ Abroad } & 1,803 & 387 & $21.5 \%$ & $0.56 \#$ & $0.49-0.63$ & 0.87 & $0.74-1.02$ \\
\hline \multicolumn{3}{|c|}{ Unknown } & 1,330 & 351 & $26.4 \%$ & $0.73 \#$ & $0.64-0.83$ & 0.97 & $0.83-1.14$ \\
\hline \multicolumn{10}{|l|}{ Age group } \\
\hline \multicolumn{3}{|c|}{$<25$ years } & 1,216 & 523 & $43.0 \%$ & (ref) & & (ref) & \\
\hline \multicolumn{3}{|c|}{$25-34$ years } & 3,443 & 1,091 & $31.7 \%$ & $0.61 \#$ & $0.54-0.70$ & 0.63\# & $0.55-0.72$ \\
\hline \multicolumn{3}{|c|}{$35-44$ years } & 2,986 & 846 & $28.3 \%$ & $0.52 \#$ & $0.46-0.60$ & $0.53 \#$ & $0.46-0.61$ \\
\hline \multicolumn{3}{|c|}{$45-54$ years } & 1,838 & 470 & $25.6 \%$ & $0.46 \#$ & $0.39-0.53$ & $0.45 \#$ & $0.39-0.53$ \\
\hline \multicolumn{3}{|c|}{$>=55$ years } & 774 & 154 & $19.9 \%$ & 0.33\# & $0.27-0.41$ & $0.34 \#$ & $0.28-0.42$ \\
\hline
\end{tabular}

${ }^{1}$ MSM men who have sex with men

${ }^{2}$ IDU persons who are intravenous drug users

${ }^{3} \mathrm{HET}$ persons with heterosexual contact

95\% Cl: 95\% Confidence interval

*OR: odds ratio

\# $p$-value: $p<=0.001$

\#\# $p$-value: $p<=0.05$

infections ranged between 15\% [34] and 25\% [35], which is lower than in those countries using the BED-CEIA for TRI. The proportion of newly diagnosed and recently infected cases is constantly low over the time compared to the high proportion of long standing and late presenting HIV infections. This is alarming from the perspective of prevention, because those who are diagnosed late are often unaware of their HIV infection for a long time and could therefore potently transmit HIV. As a consequence, early detection and diagnosis of HIV infection is of utmost importance to prevent new infections. As a strategy for eliminating infections, testing must be brought to scale, followed by immediate sustainable treatment, irrespective of the immune status. 
Men who have sex with men (MSM) are the group most at risk for HIV infection in the European Union (EU)/European Economic Association (EEA), as well as in the United States, despite targeted prevention programmes since the beginning of the HIV epidemic [36]. MSM are also the group in Germany with the highest proportion of newly diagnosed cases of recent HIV infection, similar to other European countries which used the BED-CEIA [32, 33] or other serological tests [34, 37].

It is recommended that persons at risk for HIV infection - such as MSM - should be tested at least once a year or even more often, depending on their risk behaviour $[38,39]$. This may result in a higher proportion of recently diagnosed HIV infections. It is known that the awareness, knowledge and perceived level of personal risk for HIV infection among MSM is high, which might be one reason for regular test seeking and the high proportion of recent HIV infection we found in this group. Even though they test more regularly than do other groups with risky behaviour, the proportion of recent HIV infections among MSM should be much higher. In a recent German MSM online survey, approximately $60 \%$ of the questioned MSM were tested within the previous 12 months, and approximately 53\% said they are getting tested on a regular basis [40]. However, nearly two-thirds (65\%) of MSM who were newly diagnosed with HIV were diagnosed later than 6 months after the infection occurred. Reasons for late diagnosis may vary among different groups. The fear of stigmatization, a large geographical distance to the next anonymous testing site, or a lack of risk awareness may be related to HIV testing behaviour, especially among MSM [40]. The lack of risk awareness might also be the reason for the low proportion of recent HIV infections among HET. To improve testing frequency in groups with risky behaviour, such as MSM, IDU or HET, prevention programmes should include the importance of regular and easy access to HIV testing sites and increase the awareness of risks.

Female intravenous drug users also showed a high proportion of newly diagnosed cases with recent HIV infection. We also found that female drug users of German origin had a higher chance than MSM for having a recent HIV infection. The high proportion of recent HIV infections might be related to the fact that women more often seek contact with the health care system or are better reached by testing offers for HIV or Hepatitis C during opioid substitution therapy. However, as the numbers in this sub-group were small due to the small group size, we believe that the result of the logistic regression may be related to statistical chance, and the associated results must be interpreted carefully and should be further observed in the following years. Nevertheless, this result shows the importance of adapted prevention programmes. All persons with risky sexual behaviour should be offered an HIV test regularly, whenever they have contact with the health care system. However, an HIV test should not only be offered by contact with the health care system, e.g. HIV/STI outreach clinics, it also should be included in harm reduction programmes for drug users and offered in low threshold drug services. Despite the continuous roll-out of HIV testing campaigns, services still need to improve the offers and the facilities for HIV testing to reduce late presentation.

The proportion of recent HIV infections decreased with age, meaning that long-standing infections were found more often in older age groups. This trend was also observed in other studies [33, 34, 37, 41]. The time between infection and diagnosis is usually shorter in younger persons; nevertheless, the proportion of recent HIV infection in persons older than 55 years is still nearly $24 \%$, indicating that there is ongoing HIVtransmission in older age groups.

Persons who originated from abroad more often had a long-standing infection than did German natives. This could mean that most of these cases were infected and potentially diagnosed in their home country, but the cases were also diagnosed in Germany for the first time and thus reported by the laboratories as newly diagnosed in Germany. Another explanation might be that these people did not know about their infection and that they had not been reached appropriately by prevention programmes and testing strategies in Germany. To prevent HIV infections among persons from abroad, the prevention programmes have to be tailored in a way to reach those hard-to-reach groups. To improve understanding regarding persons originating from sub-Saharan Africa and living in Germany, a study was established in 2014 at the RKI that aimed to identify their specific needs and their knowledge about HIV in order to address sustainable prevention in this sub-group in Germany [42].

The proportion of recent infections among newly diagnosed cases increased over the time period. This could suggest a real increase in HIV infections. However, this increase is not reflected by the total number of newly diagnosed infections, which remained fairly stable over the past years [31]. Therefore, the increase in recent infections could reflect more specific testing during that time period. In Germany, data are only available regarding HIV diagnoses but not regrading negative test results or the total number of HIV tests performed. It is therefore impossible to draw firm conclusions on rates solely based on recency testing among newly diagnosed HIV cases without knowledge of the testing rates.

\section{Limitations}

There are some limitations to our analysis. First, the interpretation of the proportion of recent infections among 
newly diagnosed HIV cases depends on testing patterns and the number of persons tested for HIV. There is only limited information available about the total number of persons tested in Germany. A study among HIV diagnostic laboratories estimated that approximately 1,6 million HIV screening tests were performed in 2011 [43]. In that study, the participating laboratories could not distinguish between performed tests and persons screened, and the study only had information about performed tests for one year. Interpreting time trends for HIV recency in Germany is therefore difficult. The HIV test-seeking pattern might vary between different populations; for example, MSM are tested more regularly and more frequently than other populations. Therefore, a direct estimation of the HIV incidence is not possible, and we can only report the proportion of recent infections among newly diagnosed sub-groups over time.

Second, the sensitivity and specificity of the BED-CEIA $(81.7 \%$; 89.1\%) [15] are quite low, while the reported false recent rates (FRR) are high (7.4\%) depending on the HIVsubtype distribution in the respective countries [44]. However, the BED-CEIA was evaluated using specimens from the German HIV-seroconverter cohort, where the date of infection was well-defined based on the laboratory diagnosis [29]. Therefore, the application of the BED assay to the German surveillance system is assumed to be valid to report the proportion of recent HIV infections among newly diagnosed HIV cases in Germany. Recently, we adapted the validation panel to an updated distribution of HIVsubtypes in Germany in order to re-calculate the FRR induced by the BED-CEIA, resulting in an FRR of $10 \%$ for Germany [45]. Because of the rather high FRR, a testing algorithm was applied using the recommendation of the ECDC [30]. For the reason that CD4 cell counts and viral load measurements are incomplete in the HIV notification system and are only reported in one-third of the notified cases, the recommended algorithm could only be used with the information about AIDS status. Therefore, the reported recent HIV infections may still be overestimated. Data to correct false long-standing test results are not available in the German HIV surveillance system; therefore, a correction is not possible. This might introduce an error into the data, because we might overestimate cases with long-standing infection. In 2016, we implemented a multi-assay algorithm, including the BED-CEIA and an avidity assay, to address this limitation.

\section{Conclusions}

In conclusion, the surveillance of recent HIV infections is a useful additional tool to monitor the HIV epidemic in Germany. It allows the observation of ongoing HIV transmission in Germany, and we identified (sub) populations at risk who can now be addressed more specifically in prevention programs, such as offering more frequent HIV-testing to those populations at risk. We could also identify factors associated with recent HIV infection, such as age, transmission group and origin. An early diagnosis is important for preventing new infections. Furthermore, the information gained through this part of the HIV surveillance programme can improve the statistical estimation models of the prevalence and incidence of HIV in Germany.

However, there is still the potential to improve the surveillance of recent HIV infections, for example, by implementing serological tests for recent infections with a higher sensitivity and specificity or to apply two serological tests in a multi-assay algorithm to achieve a more accurate proportion of recent HIV infections among the newly diagnosed HIV cases in Germany. It is also important to have a valid and accurate documentation of clinical data (such as CD4 count and virus load) to correct falsely identified recent or long-standing infections, as is recommended internationally. Additionally, to calculate the HIV incidence in Germany, it is important to include the total number of persons tested for HIV in Germany into the surveillance of recent HIV infections.

\begin{abstract}
Abbreviations
95\% Cl: 95\% confidence interval; AIDS: acquired immunodeficiency syndrome; BED-CEIA: BED-capture-enzyme immunoassay; CDC: Centers for Disease Control and Prevention; DPS: dried plasma spots; DSS: dried serum spots; ECDC: European Centre for Disease Prevention and Control;

EEA: European Economic Association; EU: European Union; FLS: false longstanding; FRR: false recent rate; HET: persons with heterosexual contacts; HIV: human immunodeficiency virus; IDU: persons who are intravenous drug users; MSM: men who have sex with men; OR: odds ratio; RITA: recent test algorithm; RKI: Robert Koch- Institute; TRI: test for recent (HIV) infections
\end{abstract}

\section{Acknowledgements}

We would like to thank all participating laboratories for sending us dried filter spots for laboratory analysis and for the continuous exchange of ideas and suggestions. All participating laboratories who agreed to publish their names are listed at http://www.rki.de/DE/Content/InfAZ/H/HIVAIDS/Studien/ InzSurv_HIV/beteiligte_Labore.html. Without them, the project would not have led to the present results. We would like to thank all participating staff at the RKI who were involved in the realization of the studies, especially the laboratory assistants for executing the BED-CEIA and the medical documentation officers for data entry and data quality control.

\section{Availability of data and materials}

The dataset supporting the conclusions of this article is available in the Zenodo repository: http://doi.org/10.5281/zenodo.260114.

\section{Funding}

The nationwide cross-sectional incidence study (2008-2010) and the "Surveillance of recent HIV infections" (2011-2014) were funded by the German Federal Ministry of Health.

\section{Authors' contributions}

$A H, R Z, C S H, B B$, and $A H a u$ analysed and interpreted the data. $A H, R Z$, $\mathrm{CSH}, \mathrm{BB}$, and AHau wrote the paper. $\mathrm{RZ}, \mathrm{OH}, \mathrm{CSH}, \mathrm{CK}$, and $\mathrm{BB}$ contributed to the conception and the design of the studies. JBF designed and planned the nationwide cross-sectional incidence study. SW implemented the BED-CEIA in Germany. CK, AHau and SW tested DSS/DPS samples for recency of infection and interpreted the test results. $\mathrm{RZ}, \mathrm{CSH}, \mathrm{AHau}, \mathrm{NB}, \mathrm{BB}, \mathrm{VB}, \mathrm{OH}$, and $\mathrm{CK}$ revised the article critically and $O H, N B, V B$, and $B B$ gave the final approval for publishing the article. All authors read and approved the final manuscript. 


\section{Ethics approval and consent to participate}

Ethical approval for the nationwide cross-sectional study was given by the ethics board at the Charité, University Medicine, Berlin, and approval was also granted by the data protection office of Germany according to the Federal Data Protection Act. The DSS/DPS samples used were residuals from routine HIV diagnostic processes; therefore, no informed consent was obtained from the patients. Furthermore, the DSS/DPS samples cannot be linked to individuals because the HIV notification system is strictly anonymous. Additionally, the BED-CEIA is only accredited for epidemiological studies and not for individual diagnosis. Therefore, no extra benefit can be obtained by informing persons about positive BED-CEIA test results.

\section{Consent for publication}

Not applicable.

\section{Competing interests}

The authors declare that they have no competing interests.

\section{Publisher's Note}

Springer Nature remains neutral with regard to jurisdictional claims in published maps and institutional affiliations.

\section{Author details}

'Department for Infectious Disease Epidemiology, Unit 34 HIV/AIDS, STI and Blood-Borne Infections, Robert Koch-Institute, Seestr.10, 13353 Berlin, Germany. ${ }^{2}$ Charité, Universitätsmedizin, Berlin, Germany. ${ }^{3}$ Department of Infectious Diseases, Unit 18 HIV and Other Retroviruses, Robert Koch-Institute, Nordufer 20, 13353 Berlin, Germany. ${ }^{4}$ Central Research Institute of Ambulatory Health Care in Germany (Zi), Berlin, Germany. ${ }^{5}$ SGS Belgium NV, Generaal De Wittelaan 19, B-2800 Mechelen, Belgium. ${ }^{6}$ Department for Infectious Disease Epidemiology, Robert Koch-Institute, Seestr.10, 13353 Berlin, Germany.

\section{Received: 26 January 2017 Accepted: 3 July 2017}

Published online: 11 July 2017

\section{References}

1. Robert Koch-Institut. Schätzung der Prävalenz und Inzidenz von HIVInfektionen in Deutschland, Stand Ende 2014 Epidemiologisches Bulletin 2015;45(2015).

2. Hamouda O. HIV/AIDS surveillance in Germany. J Acquir Immune Defic Syndr. 2003;32(Suppl 1):49-54.

3. Batzing-Feigenbaum J, Kollan C, Kuhne A, Matysiak-Klose D, GunsenheimerBartmeyer B, Hamouda O, et al. Cohort profile: the German ClinSurv HIV project - a multicentre open clinical cohort study supplementing national HIV surveillance. HIV Med. 2010;

4. Zu Knyphausen F, Scheufele R, Kucherer C, Jansen K, Somogyi S, Dupke S, et al. First line treatment response in patients with transmitted HIV drug resistance and well defined time point of HIV infection: updated results from the German HIV-1 seroconverter study. PLoS One. 2014;9(5):e95956.

5. Robert Koch-Institut. Weiterführende Analysen zur HIV-Inzidenz- und -Prävalenzschätzung 2012. Epidemiologisches Bulletin. 2013;45:457-64.

6. Janssen RS, Satten GA, Stramer SL, Rawal BD, O'Brien TR, Weiblen BJ, et al. New testing strategy to detect early HIV-1 infection for use in incidence estimates and for clinical and prevention purposes. JAMA. 1998;280(1):42-8.

7. Murphy G, Parry JV. Assays for the detection of recent infections with human immunodeficiency virus type 1. Euro Surveill. 2008; 13(36).

8. Guy R, Gold J, Calleja JM, Kim AA, Parekh B, Busch M, et al. Accuracy of serological assays for detection of recent infection with HIV and estimation of population incidence: a systematic review. Lancet Infect Dis. 2009;9(12):747-59.

9. Busch MP, Pilcher CD, Mastro TD, Kaldor J, Vercauteren G, Rodriguez W, et al. Beyond detuning: 10 years of progress and new challenges in the development and application of assays for HIV incidence estimation. AIDS (London, England). 2010;24(18):2763-71.

10. Curtis KA, Kennedy MS, Charurat M, Nasidi A, Delaney K, Spira TJ, et al. Development and characterization of a bead-based, multiplex assay for estimation of recent HIV type 1 infection. AIDS Res Hum Retrovir. 2012; 28(2):188-97.
11. Thomas HI, Wilson S, O'Toole CM, Lister CM, Saeed AM, Watkins RP, et al. Differential maturation of avidity of lgG antibodies to gp41, p24 and p17 following infection with HIV-1. Clin Exp Immunol. 1996;103(2):185-91.

12. Kothe D, Byers RH, Caudill SP, Satten GA, Janssen RS, Hannon WH, et al. Performance characteristics of a new less sensitive HIV-1 enzyme immunoassay for use in estimating HIV seroincidence. J Acquir Immune Defic Syndr. 2003;33(5):625-34

13. Rawal BD, Degula A, Lebedeva L, Janssen RS, Hecht FM, Sheppard HW, et al. Development of a new less-sensitive enzyme immunoassay for detection of early HIV-1 infection. J Acquir Immune Defic Syndr. 2003;33(3):349-55.

14. Barin F, Meyer L, Lancar R, Deveau C, Gharib M, Laporte A, et al. Development and validation of an immunoassay for identification of recent human immunodeficiency virus type 1 infections and its use on dried serum spots. J Clin Microbiol. 2005;43(9):4441-7.

15. Parekh BS, Kennedy MS, Dobbs T, Pau CP, Byers R, Green T, et al. Quantitative detection of increasing HIV type 1 antibodies after seroconversion: a simple assay for detecting recent HIV infection and estimating incidence. AIDS Res Hum Retrovir. 2002;18(4):295-307.

16. Suligoi B, Galli C, Massi M, Di Sora F, Sciandra M, Pezzotti P, et al. Precision and accuracy of a procedure for detecting recent human immunodeficiency virus infections by calculating the antibody avidity index by an automated immunoassay-based method. J Clin Microbiol. 2002;40(11):4015-20.

17. Masciotra S, Dobbs T, Candal D, Hanson D, Delaney K, editors. Antibody avidity-based assay for identifying recent HIV-1 infections Based on Genetic Systems [TM] 1/2 Plus O EIA2010 16.-19.2.2010 17th Conference on Retroviruses and Opportunistic Infections.

18. Wei X, Liu X, Dobbs T, Kuehl D, Nkengasong JN, Hu DJ, et al. Development of two avidity-based assays to detect recent HIV type 1 seroconversion using a multisubtype gp41 recombinant protein. AIDS Res Hum Retrovir. 2010;26(1):61-71.

19. Suligoi B, Rodella A, Raimondo M, Regine V, Terlenghi L, Manca N, et al. Avidity index for anti-HIV antibodies: comparison between third- and fourthgeneration automated immunoassays. J Clin Microbiol. 2011;49(7):2610-3.

20. Chawla A, Murphy G, Donnelly C, Booth CL, Johnson M, Parry JV, et al. Human immunodeficiency virus (HIV) antibody avidity testing to identify recent infection in newly diagnosed HIV type 1 (HIV-1)-seropositive persons infected with diverse HIV-1 subtypes. J Clin Microbiol. 2007;45(2):415-20.

21. Hu DJ, Vanichseni S, Mock PA, Young NL, Dobbs T, Byers RH Jr, et al. HIV type 1 incidence estimates by detection of recent infection from a cross-sectional sampling of injection drug users in Bangkok: use of the lgG capture BED enzyme immunoassay. AIDS Res Hum Retrovir. 2003;19(9):727-30.

22. Kim AA, McDougal JS, Hargrove J, Rehle T, Pillay-Van Wyk V, Puren A, et al. Evaluating the BED capture enzyme immunoassay to estimate HIV incidence among adults in three countries in sub-Saharan Africa. AIDS Res Hum Retrovir. 2010;26(10):1051-61.

23. Scheer $S$, Nakelsky S, Bingham T, Damesyn M, Sun D, Chin CS, et al. Estimated HIV incidence in California, 2006-2009. PLoS One. 2013;8(2): e55002.

24. Bätzing-Feigenbaum J, Loschen S, Gohlke-Micknis S, Hintsche B, Rausch M, Hillenbrand $\mathrm{H}$, et al. Implications of and perspectives on HIV surveillance using a serological method to measure recent HIV infections in newly diagnosed individuals: results from a pilot study in berlin, Germany, in 2005\&2007. HIV Medicine. 2009;10(4):209-18.

25. Batzing-Feigenbaum J, Loschen S, Gohlke-Micknis S, Zimmermann R, Herrmann A, Kamga Wambo O, et al. Country-wide HIV incidence study complementing HIV surveillance in Germany. Euro Surveill. 2008;13(36).

26. Santos-Hovener C, Zimmermann R, Kucherer C, Batzing-Feigenbaum J, Wildner $\mathrm{S}$, Hamouda $\mathrm{O}$, et al. Conversation about Serostatus decreases risk of acquiring HIV: results from a case control study comparing MSM with recent HIV infection and HIV negative controls. BMC Public Health. 2014;14:453.

27. Calypte. Aware ${ }^{\text {TM }}$ BED ${ }^{\text {TM }}$ EIA HIV-1 Incidence Test (IgG-Capture HIV-EIA). Enzyme Immunoassay for Population Esitmates of HIV-1 Incidence. Portland, USA: Calypte Biomedical Corporation; 2008.

28. Sedia Biosciences Corporation. Sedia ${ }^{T M}$ BED HIV-1 Incidence EIA. Enzyme Immunoassay for Population Esitmates of HIV-1 Incidence. Portland, USA: Sedia Biosciences Corporation; 2014.

29. Loschen S, Batzing-Feigenbaum J, Poggensee G, Cordes C, Hintsche B, Rausch $\mathrm{M}$, et al. Comparison of the human immunodeficiency virus (HIV) type 1-specific immunoglobulin G capture enzyme-linked immunosorbent assay and the avidity index method for identification of recent HIV infections. J Clin Microbiol. 2008;46(1):341-5. 
30. European Centre for Disease Prevention and Control. Monitoring recently acquired HIV infections in the European context. Stockholm: ECDC; 2013 [cited 2016 Jan 25]. Available from: http:/www.ecdc.europa.eu/en/ publications/Publications/monitoring-recently-acquired-HIV-infectionseuropean-context.pdf.

31. Robert Koch-Institut. HIV-Diagnosen und AIDS-Erkrankungen in Deutschland: Bericht zur Entwicklung im Jahr 2014 aus dem Robert Koch-Institut. Epidemiologisches Bulletin. 2015;27(2015).

32. Romero A, Gonzalez V, Esteve A, Martro E, Matas L, Tural C, et al. Identification of recent HIV-1 infection among newly diagnosed cases in Catalonia, Spain (2006-08). Eur J Pub Health. 2012;22(6):802-8.

33. Widgren $\mathrm{K}$, Skar H, Berglund T, Kling AM, Tegnell A, Albert J. Delayed HIV diagnosis common in Sweden, 2003-2010. Scand J Infect Dis. 2014;46(12):862-7.

34. Aghaizu A, Murphy G, Tosswill J, DeAngelis D, Charlett A, Gill ON, et al. Recent infection testing algorithm (RITA) applied to new HIV diagnoses in England, Wales and Northern Ireland, 2009 to 2011. Euro Surveill. 2014;19(2).

35. Le Vu S, Le Strat Y, Barin F, Pillonel J, Cazein F, Bousquet V, et al. Populationbased HIV-1 incidence in France, 2003-08: a modelling analysis. Lancet Infect Dis. 2010;10(10):682-7.

36. De Cock KM, Jaffe HW, Curran JW. The evolving epidemiology of HIV/AIDS AIDS (London, England). 2012;26(10):1205-13.

37. Semaille C, Cazein F, Pillonel J, Lot F, Le Vu S, Pinget R, et al. Four years of surveillance of recent HIV infections at country level, France, mid 20032006: experience and perspectives. Euro Surveill. 2008:13(36).

38. Gokengin D, Geretti AM, Begovac J, Palfreeman A, Stevanovic M, Tarasenko O, et al. 2014 European guideline on HIV testing. Int J STD AIDS. 2014; 25(10):695-704.

39. Arbeitsgemeinschaft der Wissenschaftlichen Medizinischen Fachgesellschaften, Deutsche STI-Gesellschaft, Robert Koch-Institut, Bundeszentrale für gesundheitsliche Aufklärung, Landeszentrum Gesundheit Nordrgein-Westfalen. [S1 Guidline: STI/STD- Counselling, Diagnostic and Therapy] 2015 [cited 2016 Jan 25]. Available from: http://www.awmf.org/ uploads/tx_szleitlinien/059-006l_S1_STI_STD-Beratung_2015-07.pdf.

40. Marcus U, Gassowski M, Kruspe M, Drewes J. Recency and frequency of HIV testing among men who have sex with men in Germany and sociodemographic factors associated with testing behaviour. BMC Public Health. 2015;15:727

41. Romero A, Gonzalez V, Granell M, Matas L, Esteve A, Martro E, et al. Recently acquired HIV infection in Spain (2003-2005): introduction of the serological testing algorithm for recent HIV seroconversion. Sex Transm Infect. 2009; 85(2):106-10.

42. Robert Koch-Institut. [Knowledge Attitude Behavior Study about HIV and STI Testing in Germany for migrants from Sub-Saharan-Africa (MISSA)] Berlin: Robert Koch-Institut; 2015 [cited 2016 Feb 25]. Available from: http://www. rki.de/DE/Content/InfAZ/H/HIVAIDS/Studien/MiSSA/MiSSA_node.html.

43. Robert Koch-Institut. HIV-Nennerstudie: Online Laborbefragung zur Bestandsaufnahme der HIV-Diagnostik in Deutschland 2011. Epidemiologisches. Bulletin. 2015;07(15):47-50

44. Kassanjee R, Pilcher CD, Keating SM, Facente SN, McKinney E, Price MA, et al. Independent assessment of candidate HIV incidence assays on specimens in the CEPHIA repository. AIDS (London, England). 2014;28(16): 2439-49.

45. Hauser A, Hofmann A, Meixenberger K, Somogyi S, Bartmeyer B, Jansen K, et al., editors. Comparing a 'Multi Assay Algorithm' with the BED Capture Enzyme Immunoassay for the testing of recent HIV-1 infections in Germany. 15th European AIDS Conference; 2015 21.10-24.10.2015; Barcelona, Spain.

\section{Submit your next manuscript to BioMed Central and we will help you at every step:}

- We accept pre-submission inquiries

- Our selector tool helps you to find the most relevant journal

- We provide round the clock customer support

- Convenient online submission

- Thorough peer review

- Inclusion in PubMed and all major indexing services

- Maximum visibility for your research

Submit your manuscript at www.biomedcentral.com/submit

CBiomed Central 